IFT-10/2010

SACLAY-T10/094

\title{
Higgs as a pseudo-Goldstone boson, the mu problem and gauge-mediated supersymmetry breaking
}

\author{
Anna Kamińska $^{a}$ and Stéphane Lavignac ${ }^{b}$ \\ ${ }^{a}$ Institute of Theoretical Physics, Faculty of Physics, \\ University of Warsaw, Hoża 69, Warsaw, Poland \\ ${ }^{b}$ Institut de Physique Théorique, CEA-Saclay, \\ F-91191 Gif-sur-Yvette Cedex, France 1
}

\begin{abstract}
We study the interplay between the spontaneous breaking of a global symmetry of the Higgs sector and gauge-mediated supersymmetry breaking, in the framework of a supersymmetric model with global $S U(3)$ symmetry. In addition to solving the supersymmetric flavour problem and alleviating the little hierarchy problem, this scenario automatically triggers the breaking of the global symmetry and provides an elegant solution to the $\mu / B \mu$ problem of gauge mediation. We study in detail the processes of global symmetry and electroweak symmetry breaking, including the contributions of the top/stop and gauge-Higgs sectors to the one-loop effective potential of the pseudo-Goldstone Higgs boson. While the joint effect of supersymmetry and of the global symmetry allows in principle the electroweak symmetry to be broken with little fine-tuning, the simplest version of the model fails to bring the Higgs mass above the LEP bound due to a suppressed tree-level quartic coupling. To cure this problem, we consider the possibility of additional $S U(3)$-breaking contributions to the Higgs potential, which results in a moderate fine-tuning. The model predicts a rather low messenger scale, a small $\tan \beta$ value, a light Higgs boson with Standard Model-like properties, and heavy higgsinos.
\end{abstract}

\section{Introduction}

Among the proposed extensions of the Standard Model, supersymmetry is one of the most attractive from a theoretical point of view, in particular because it automatically solves the hierarchy problem. However, the lack of experimental evidence put strong constraints on supersymmetric models such as the Minimal Supersymmetric Standard Model (MSSM). The fact that no superpartner has been discovered so far implies a (at least in part) heavy supersymmetric spectrum, which exacerbates the "little hierarchy" problem associated with the LEP bound on the Higgs mass and increases the level of fine-tuning in the Higgs potential. Furthermore, the absence of any significant deviation from the Standard Model predictions in flavour physics places strong restrictions on the generational structure of squark and slepton masses. Gauge mediation [1] offers a natural solution to this problem: supersymmetry breaking is communicated to the observable sector by gauge interactions, and is therefore automatically flavour blind. On the other hand, gauge mediation suffers from the so-called $\mu / B \mu$ problem [2], i.e. the fact that the $\mu$

\footnotetext{
${ }^{1}$ Laboratoire de la Direction des Sciences de la Matière du Commissariat à l'Energie Atomique et Unité de Recherche Associée au CNRS (URA 2306).
} 
and $B \mu$ parameters of the MSSM are typically generated at the same loop order, leading to $B \mu \gg|\mu|^{2}$, which is inconsistent with natural electroweak symmetry breaking.

Both problems - the little hierarchy problem and the $\mu / B \mu$ problem of gauge mediation - may actually have a common solution in terms of pseudo-Goldstone bosons. In Ref. [2], a mechanism involving additional singlet superfields was proposed to generate the $\mu$ and $B \mu$ parameters at the one- and two-loop levels, respectively, leading to the order-of-magnitude relation $B \mu \sim|\mu|^{2}$. It was pointed out that this relation has a pseudo-Goldstone interpretation: in some limit where the Higgs superpotential becomes invariant under a global $S U(3)$ symmetry, one combination of the two Higgs doublets remains massless after spontaneous breaking of this symmetry due to the relation $B \mu=|\mu|^{2}$ (in which the soft Higgs mass parameters have been omitted). Regarding the little hierarchy problem, it is well known that it can be alleviated if the Higgs boson arises as the pseudo-Goldstone boson of some spontaneously broken approximate global symmetry, a scenario known as little Higgs [3]. It was shown in Refs. [4, 5, 6, 7] that the combination of supersymmetry and of a global symmetry leads to an improved protection of the Higgs potential. Namely, the logarithmic dependence of the MSSM Higgs mass parameter on the cut-off scale is replaced by a dependence on the scale of spontaneous global symmetry breaking, thus reducing the need for a fine-tuning. Explicit realizations of this idea of double protection [5] of the Higgs potential by supersymmetry and by a global symmetry have shown that a fine-tuning smaller than $10 \%$ can be achieved with squark masses around $1 \mathrm{TeV}$.

In this paper, we revisit these issues by combining gauge-mediated supersymmetry breaking with a spontaneously broken global symmetry in the Higgs sector. We consider a simple model with a global $S U(3)$ symmetry, which is essentially a gauge-mediated version of the model of Ref. [5], and study it in detail. We pay particular attention to the spontaneous breaking of the global symmetry, and check whether the $S U(3)$-breaking minimum is the global minimum of the scalar potential. A key role in the process of global symmetry breaking is played by the tadpole of an $S U(3)$ singlet field, which is generated by loops of messenger fields together with the singlet soft terms. This tadpole also triggers the generation of the $\mu$ and $B \mu$ parameters; however they do not contribute to the potential of the lightest Higgs doublet due to its (pseudo-) Goldstone boson nature. As a result, the higgsinos and the non-Goldstone Higgs scalars can be made heavy with masses of order $|\mu| \gg M_{Z}$ without introducing a strong fine-tuning in the Higgs potential. We then compute the one-loop effective potential of the pseudo-Goldstone Higgs doublet and study electroweak symmetry breaking. While the corrections to the pseudoGoldstone mass parameter are efficiently controlled by the joint effect of supersymmetry and of the global symmetry, allowing in principle the electroweak symmetry to be broken with little fine-tuning, the specific model studied in this paper fails to bring the Higgs mass above the LEP bound due to a suppressed tree-level quartic coupling. To cure this problem, we consider the possibility of additional $S U(3)$-breaking contributions to the Higgs potential, and estimate the resulting fine-tuning.

The paper is organized as follows. In Section 2, we describe the model and discuss the generation of the soft supersymmetry breaking terms via gauge mediation. In Section 3, we study the spontaneous symmetry breaking of the global $S U(3)$ symmetry and find a region of the parameter space in which the desired vacuum is indeed the global minimum of the scalar potential. We then show how the $\mu / B \mu$ problem is solved by the global symmetry. Section 4 deals with electroweak symmetry breaking, the Higgs mass and fine-tuning. Finally, we give our conclusions in Section 5 ,

\section{The model}

The model we study in this letter is based on a supersymmetric version [5] of the "simplest little Higgs model" of Ref. [8]. We describe its general structure below, before discussing the 
generation of the soft supersymmetry breaking terms via gauge mediation.

In order to realize the Higgs as pseudo-Goldstone boson idea, a global $S U(3)$ symmetry spontaneously broken at the scale $f \sim 1 \mathrm{TeV}$ is imposed on the Higgs sector. The MSSM Higgs doublets $H_{d}$ and $H_{u}$ are extended to global $S U(3)$ (anti-)triplets:

$$
\mathcal{H}_{d}=\left(\begin{array}{c}
H_{d} \\
S_{d}
\end{array}\right) \in \mathbf{3}, \quad \mathcal{H}_{u}^{T}=\left(\begin{array}{c}
i \sigma^{2} H_{u} \\
S_{u}
\end{array}\right) \in \overline{\mathbf{3}},
$$

where $S_{u}$ and $S_{d}$ are electroweak singlets. Similarly, all matter fields are extended to $S U(3)$ multiplets. The global symmetry is spontaneously broken by the VEVs of $S_{u}$ and $S_{d}$. The associated Goldstone boson, which is identified with the Standard Model Higgs boson $H$, is a linear combination of the $S U(2)_{L}$ doublets $H_{d}$ and $i \sigma^{2} H_{u}^{*}$. By construction, the tree-level Higgs potential does not contain a mass term for $H$. However, the global $S U(3)$ symmetry of the Higgs sector is not a symmetry of the full Lagrangian: it is violated explicitly by the Yukawa and gauge interactions of the MSSM. These induce a one-loop potential for $H$ which, due to the combined effect of supersymmetry and of the approximate global $S U(3)$ symmetry, has no logarithmic dependence on the ultraviolet cut-off [5]. Thanks to this softening of the radiative corrections, the LEP Higgs mass bound can be satisfied with less fine-tuning than in the MSSM.

In order for this double protection mechanism to be operative, the gauge symmetry must be compatible with the global symmetry in the ultraviolet. To this end, the electroweak gauge symmetry $S U(2)_{L} \times U(1)_{Y}$ is extended to $S U(3)_{W} \times U(1)_{X}$, where $Y=X-T^{8} / \sqrt{3}$. The breaking of the extended gauge group is achieved at some higher energy scale $F \gg f$ by two additional Higgs (anti-)triplets $\Phi_{D}$ and $\Phi_{U}$. In this way, the masses of the heavy gauge bosons are unrelated to the global symmetry breaking scale $f$, and experimental limits on them do not constrain it. It is then possible to choose $f$ around the $\mathrm{TeV}$ scale, so as to minimize the finetuning in the Higgs potential, without running into conflict with precision electroweak data [5].

The details of the model are presented below.

\subsection{The $S U(3)$-symmetric Higgs sector}

The Higgs sector has a global $S U(3)_{1} \times S U(3)_{2}$ symmetry whose diagonal subgroup is the $S U(3)_{W}$ gauge symmetry. It contains the following Higgs multiplets:

- $\Phi_{D}$ and $\Phi_{U}$, transforming as $\mathbf{3}$ and $\overline{\mathbf{3}}$ of $S U(3)_{1}$,

- $\mathcal{H}_{d}$ and $\mathcal{H}_{u}$, transforming as $\mathbf{3}$ and $\overline{\mathbf{3}}$ of $S U(3)_{2}$,

- two $S U(3)_{1} \times S U(3)_{2}$ singlets $N$ and $N^{\prime}$.

Under $S U(3)_{W} \times U(1)_{X}, \Phi_{D}$ and $\mathcal{H}_{d}\left(\Phi_{U}\right.$ and $\left.\mathcal{H}_{u}\right)$ have quantum numbers $\mathbf{3}_{-1 / 3}\left(\overline{\mathbf{3}}_{+1 / 3}\right)$, while $N$ and $N^{\prime}$ are singlets. The MSSM Higgs doublets $H_{d}$ and $H_{u}$ are embedded in $\mathcal{H}_{d}$ and $\mathcal{H}_{u}$ as indicated in Eq. (11). The $S U(3)_{1} \times S U(3)_{2}$ symmetric Higgs superpotential is chosen to be:

$$
W_{\mathrm{Higgs}}=\lambda^{\prime} N^{\prime}\left(\Phi_{U} \Phi_{D}-\frac{F^{2}}{2}\right)+\lambda N \mathcal{H}_{u} \mathcal{H}_{d}+\frac{\kappa}{3} N^{3},
$$

where the last two terms are reminiscent of the NMSSM [9]. The role of the $N \mathcal{H}_{u} \mathcal{H}_{d}$ coupling is to induce the breaking of the global $S U(3)_{2}$ symmetry once the singlet field $N$ acquires a VEV. This coupling is also responsible, as in the NMSSM, for the generation of the $\mu$ and $B \mu$ parameter through the VEVs of the scalar and F-term components of $N$. The last term is crucial to avoid a runaway of the tree-level scalar potential in the $N$ direction. 
The superpotential (21) leads to the spontaneous breaking of the global $S U(3)_{1}$ symmetry, together with the gauge symmetry breaking $S U(3)_{W} \times U(1)_{X} \rightarrow S U(2)_{L} \times U(1)_{Y}$ :

$$
\left\langle\Phi_{D}\right\rangle=\left(\begin{array}{c}
0 \\
0 \\
F_{D}
\end{array}\right), \quad\left\langle\Phi_{U}\right\rangle=\left(\begin{array}{ccc}
0 & 0 & F_{U}
\end{array}\right),
$$

with $F_{U}=F_{D}=F / \sqrt{2}$ in the supersymmetric limit (soft terms will shift the VEVs of $\Phi_{D}$ and $\Phi_{U}$ by an amount $\left.\mathcal{O}\left(m_{\text {soft }}^{2} / F\right)\right)$. The spontaneous breaking of the global $S U(3)_{2}$ symmetry is triggered by a tadpole term in the singlet field $N$, whose origin will be discussed in Section 2.3.

$$
\left\langle\mathcal{H}_{d}\right\rangle=\left(\begin{array}{c}
0 \\
0 \\
f \cos \beta
\end{array}\right), \quad\left\langle\mathcal{H}_{u}\right\rangle=\left(\begin{array}{lll}
0 & 0 & f \sin \beta
\end{array}\right),
$$

where we have defined $2 \tan \beta \equiv\left\langle S_{u}\right\rangle /\left\langle S_{d}\right\rangle$.

One can take advantage of the hierarchy $F \gg f$ to integrate out at the scale $F$ the heavy components of the chiral superfields $\Phi_{D}, \Phi_{U}$ and $N^{\prime}$, as well as the heavy gauge supermultiplets living in the coset $S U(3)_{W} \times U(1)_{X} / S U(2)_{L} \times U(1)_{Y}$. The resulting effective field theory is then used to study the breaking of the global $S U(3)_{2}$ symmetry and of the electroweak symmetry.

\subsection{The top quark sector}

Like the Higgs fields, the matter fields of the MSSM must be extended to $S U(3)$ multiplets. Since we are mostly interested in electroweak symmetry breaking, we only need to consider the top/stop sector, which gives the dominant contribution to the one-loop effective potentia 3 . For definiteness, we make the same choice as Ref. [5] for the representations of the top quark superfields and for their couplings to the Higgs superfields (see e.g. Refs. [11, 12] for alternative choices):

$$
W_{\text {top }}=y_{1} \Phi_{U} \Psi_{Q} T^{c}+y_{2} \mathcal{H}_{u} \Psi_{Q} t^{c},
$$

where $\Psi_{Q}=\left(Q^{T}, T\right)^{T}=((t, b), T)^{T}$ is an $S U(3)_{W}$ triplet, while $t^{c}$ and $T^{c}$ are $S U(3)_{W}$ singlets (obviously, a second singlet is necessary to render both the top quark and its $S U(3)_{W}$ partner $T$ massive). Below the scale $F$, the first coupling in Eq. (5) is replaced by the effective mass term $y_{1} F T T^{c}$. The simultaneous presence of the two terms violates the global $S U(3)_{2}$ symmetry; hence all $S U(3)_{2}$-breaking effects from the top/stop sector will be proportional to $y_{1} y_{2}$.

\subsection{Soft supersymmetry breaking terms}

So far the model described above is identical to the one of Ref. [5]. The difference lies in the soft supersymmetry breaking terms, which in our model are calculable in terms of a few parameters. Namely, we assume that supersymmetry is broken in a secluded sector and communicated to the observable sector via gauge interactions. As is customary, we parameterize supersymmetry breaking by a gauge-singlet spurion superfield $X$ and couple it to a vector-like pair of chiral messenger superfields $(\Phi, \bar{\Phi})$, which we choose to be in the representation $\left(\overline{\mathbf{3}}, \mathbf{1}, \frac{1}{3}\right) \oplus\left(\mathbf{1}, \mathbf{3},-\frac{1}{3}\right)$ of $S U(3)_{C} \times S U(3)_{W} \times U(1)_{X}$ and its conjugate. In order to generate soft terms for the singlet superfield $N$, we also introduce a coupling $N \bar{\Phi} \Phi$ :

$$
W_{\text {mess }}=X \bar{\Phi} \Phi+\xi N \bar{\Phi} \Phi, \quad\langle X\rangle=M+\theta^{2} F_{X} .
$$

\footnotetext{
${ }^{2}$ This notation, which is reminiscent of the one used in the MSSM for the ratio of the two Higgs doublet VEVs, is motivated by the fact that the pseudo-Goldstone boson is given by the same linear combination $H \simeq$ $\cos \beta H_{d}+\sin \beta\left(i \sigma^{2} H_{u}^{*}\right)$ as the lightest MSSM Higgs boson in the decoupling regime [10].

${ }^{3}$ As we are going to see, $\tan \beta$ turns out to be small in this model, so that the bottom/sbottom contribution to the one-loop effective potential can be neglected.
} 
The soft supersymmetry breaking terms for the gauginos and gauge non-singlet scalars are generated by the standard messenger loops [1], and are schematically given by (the explicit formulae can be found in Appendix (B):

$$
m_{\text {gaugino }} \sim \frac{\alpha}{4 \pi} \Lambda, \quad m_{\text {scalar }}^{2} \sim\left(\frac{\alpha}{4 \pi}\right)^{2} \Lambda^{2}, \quad \Lambda \equiv \frac{F_{X}}{M} .
$$

These expressions are valid at the messenger scale $M$, with $\alpha=g^{2}(M) / 4 \pi$, where $g(\mu)$ is the relevant running gauge coupling. The $A$-terms associated with the Yukawa couplings of the top sector, $A_{y_{1}}$ and $A_{y_{2}}$, vanish at the messenger scale and are generated at lower scales by renormalization group running. Due to the direct coupling between $N$ and the messenger fields, soft terms for the gauge-singlet superfield $N$ are also generated (by contrast, the soft terms for the singlet $N^{\prime}$ vanish). Using the wave-function renormalization technique of Ref. [13], we find:

$$
\begin{gathered}
A_{\lambda}=\frac{A_{\kappa}}{3}=-\frac{6 \xi^{2}}{16 \pi^{2}} \Lambda, \\
m_{N}^{2}=\frac{1}{\left(16 \pi^{2}\right)^{2}}\left(48 \xi^{4}-24 \kappa^{2} \xi^{2}-16 g_{C}^{2} \xi^{2}-16 g_{W}^{2} \xi^{2}-\frac{8}{3} g_{X}^{2} \xi^{2}\right) \Lambda^{2},
\end{gathered}
$$

where $g_{C}, g_{W}$ and $g_{X}$ are the $S U(3)_{C}, S U(3)_{W}$ and $U(1)_{X}$ gauge couplings, respectively. Note that $m_{N}^{2}<0$ as soon as $\xi \lesssim g_{W}$. A negative contribution to the soft masses of the Higgs triplets $\mathcal{H}_{d}$ and $\mathcal{H}_{u}$ is also induced by the $\xi$ coupling, on top of the standard (positive) gauge mediation contribution:

$$
m_{u}^{2}=m_{d}^{2}=\frac{1}{\left(16 \pi^{2}\right)^{2}}\left(\frac{8}{3} g_{W}^{4}+\frac{8}{27} g_{X}^{4}-6 \lambda^{2} \xi^{2}\right) \Lambda^{2} .
$$

Last but not least, the presence of a direct coupling between the singlet $N$ and the messenger superfields also induces a tadpole in the scalar potential [14, 2, 15]:

$$
V_{\text {tad }}=m^{3} N+\text { h.c. }, \quad m^{3}=\frac{6 \xi}{16 \pi^{2}} \Lambda^{2} M,
$$

which plays an essential role in the breaking of the global $S U(3)_{2}$ symmetry, as well as an effective tadpole term in the superpotential [14, 15]:

$$
W_{\mathrm{tad}}=M_{N}^{2} N, \quad M_{N}^{2} \sim \frac{6 \xi}{16 \pi^{2}} F_{X}^{*} .
$$

Let us note in passing that we could have avoided the generation of a tadpole for $N$ by introducing a second pair of messenger fields with the following superpotential couplings [13, 16]:

$$
W_{\text {mess }}=X \bar{\Phi}_{1} \Phi_{1}+X \bar{\Phi}_{2} \Phi_{2}+\xi N \bar{\Phi}_{1} \Phi_{2} .
$$

Since $X$ and $N$ couple to different combinations $\Phi_{i} \bar{\Phi}_{j}$, no tadpole arises at one loop and the breaking of the global $S U(3)_{2}$ symmetry is triggered by the singlet soft terms (with $m_{N}^{2}<0$ for $\xi \lesssim g_{W}$ ). In this letter, we choose to stick to the minimal case involving a single pair of messenger fields, with the superpotential (6).

\section{Spontaneous breaking of the global $S U(3)_{2}$ symmetry}

In order to proceed with the analysis of the global $S U(3)_{2}$ symmetry breaking, it is convenient to integrate out the fields that acquire a mass of order $F$ when the gauge symmetry $S U(3)_{W} \times U(1)_{X}$ breaks down to $S U(2)_{L} \times U(1)_{Y}$. One is then left with the following tree-level potential for the fields $N, \mathcal{H}_{d}$ and $\mathcal{H}_{u}$, valid for energy scales $E \ll F$ :

$$
V_{\mathrm{Higgs}}=V_{F}+V_{D}+V_{\mathrm{soft}}+\delta V_{\mathrm{soft}}+V_{\mathrm{tad}},
$$




$$
\begin{gathered}
V_{F}=\left|\lambda \mathcal{H}_{u} \mathcal{H}_{d}+\kappa N^{2}+M_{N}^{2}\right|^{2}+|\lambda|^{2}|N|^{2}\left(\left|\mathcal{H}_{u}\right|^{2}+\left|\mathcal{H}_{d}\right|^{2}\right) \\
V_{D}=\frac{g^{2}}{8} \sum_{i}\left(H_{u}^{\dagger} \sigma^{i} H_{u}+H_{d}^{\dagger} \sigma^{i} H_{d}\right)^{2}+\frac{g^{\prime 2}}{8}\left(\left|H_{u}\right|^{2}-\left|H_{d}\right|^{2}\right)^{2} \\
V_{\text {soft }}=m_{N}^{2}|N|^{2}+m_{u}^{2}\left|\mathcal{H}_{u}\right|^{2}+m_{d}^{2}\left|\mathcal{H}_{d}\right|^{2}+\left(\lambda A_{\lambda} N \mathcal{H}_{u} \mathcal{H}_{d}+\frac{\kappa}{3} A_{\kappa} N^{3}+\text { h.c. }\right), \\
\delta V_{\text {soft }}=\left(m_{D}^{2}-m_{U}^{2}\right)\left[\frac{9-21 t_{W}^{2}+4 t_{W}^{4}}{36}\left(H_{u}^{\dagger} H_{u}-H_{d}^{\dagger} H_{d}\right)+\frac{1}{2}\left(S_{u}^{*} S_{u}-S_{d}^{*} S_{d}\right)\right] \\
V_{\text {tad }}=m^{3} N+\text { h.c. }
\end{gathered}
$$

where $g$ and $g^{\prime}$ are the $S U(2)_{L}$ and $U(1)_{Y}$ gauge couplings $4, t_{W} \equiv g^{\prime} / g$ and the expressions for the soft terms $m_{N}^{2}, m_{u}^{2}, m_{d}^{2}, A_{\lambda}, A_{\kappa}$ and tadpole parameters $m^{3}, M_{N}^{2}$ have been given before. $V_{D}$ contains the $S U(2)_{L} \times U(1)_{Y} D$-terms and breaks the global $S U(3)_{2}$ symmetry. The term $\delta V_{\text {soft }}$, which is a residue of integrating out the heavy gauge supermultiplets [17, also breaks $S U(3)_{2}$; it is proportional to $m_{D}^{2}-m_{U}^{2}$, the difference between the soft masses squared of the $S U(3)_{1}$ Higgs triplets $\Phi_{D}$ and $\Phi_{U}[5,6]$. This term is potentially dangerous because it gives a tree-level mass to the Higgs boson, thus spoiling its pseudo-Goldstone nature. However, since $\Phi_{D}$ and $\Phi_{U}$ are in conjugate representations, they have equal soft masses at the messenger scale and the splitting $m_{D}^{2}-m_{U}^{2}$ is generated by the running between $M$ and $F$; hence it is expected to be small. Indeed, numerical calculations show that the effect of $\delta V_{\text {soft }}$ on the dynamics of $S U(3)_{2}$ breaking and on the value of the Higgs mass is negligible.

The tadpole (19) triggers a VEV $v_{N} \equiv\langle N\rangle \sim m \sim\left(6 \xi M m_{\text {soft }}^{2} / \alpha^{2}\right)^{1 / 3}$, together with $f \sim m$. On the other hand, $f \lesssim 1 \mathrm{TeV}$ is needed in order not to spoil the pseudo-Goldstone nature of the Higgs boson. This points towards a rather small value of $\xi$, of the order $\xi \lesssim$ $10^{-3} \alpha^{2}\left(1 \mathrm{TeV} / m_{\mathrm{soft}}\right)^{2}(100 \mathrm{TeV} / M)$. In practice this means that the soft terms $A_{\lambda}, A_{\kappa}$ and $m_{N}^{2}$, as well as the negative contribution to $m_{u}^{2}$ and $m_{d}^{2}$, are strongly suppressed and can be neglected in the minimization of the scalar potential. As for the superpotential tadpole term, which is of order $M_{N}^{2} \sim(6 \xi / 4 \pi) M m_{\text {soft }} / \alpha$, its only effect is to shift $v_{N}$ by a relative amount $M_{N}^{2} / m^{2} \sim(\alpha / 4 \pi) m / m_{\text {soft }} \lesssim(\alpha / 4 \pi)\left(1 \mathrm{TeV} / m_{\text {soft }}\right)$, and we will omit it in the following analytical considerations. Nevertheless all these parameters are included in our numerical computations.

While the VEV of $N$ is stabilized by the superpotential term $\kappa N^{3} / 3$, there is no term in $V$ to stabilize a VEV of $S_{u}\left(S_{d}\right)$ triggered by a negative $m_{u}^{2}\left(m_{d}^{2}\right)$. Since $m_{u}^{2}$ is driven negative by the renormalization group running between the messenger scale $M \gtrsim 100 \mathrm{GeV}$ and the gauge symmetry breaking scale $F$, this leads to a runaway in the direction $\langle N\rangle=\left\langle S_{d}\right\rangle=0,\left|\left\langle S_{u}\right\rangle\right| \rightarrow \infty$, which we discuss in the next subsection.

\subsection{Global $S U(3)_{2}$ symmetry breaking: analytical discussion}

Let us first ignore the runaway direction and minimize the scalar potential for $v_{N} \equiv\langle N\rangle \neq 0$. We have argued in the previous subsection that the soft terms associated with $N$ as well as the superpotential tadpole parameter $M_{N}^{2}$ can be neglected to a good approximation. Furthermore, for reasons that will become clear later, a tadpole parameter $m$ somewhat larger than the scale of MSSM soft terms is needed in order for the proper symmetry breaking vacuum to be the global minimum of the scalar potential. This leads to the prediction of a small $\tan \beta$, since the minimization conditions give:

$$
\tan ^{2} \beta=\frac{\lambda^{2} v_{N}^{2}+m_{d}^{2}}{\lambda^{2} v_{N}^{2}+m_{u}^{2}}
$$

\footnotetext{
${ }^{4}$ The matching conditions between the $S U(3)_{W} \times U(1)_{X}$ and $S U(2)_{L} \times U(1)_{Y}$ gauge couplings at the scale $F$ read $g=g_{W}$ and $g^{\prime}=g_{W} g_{X} / \sqrt{g_{W}^{2}+g_{X}^{2} / 3}$.
} 
together with $v_{N} \sim m$. Neglecting all soft terms (including the Higgs soft masses $m_{u}$ and $m_{d}$ ) in the minimization of the scalar potential, we obtain the following approximate solution:

$$
v_{N}^{3}=\frac{m^{3}}{2 \lambda(2 \kappa+\lambda)}, \quad f= \pm \frac{[-2(\kappa+\lambda)]^{1 / 2}}{\lambda^{5 / 6}[2(2 \kappa+\lambda)]^{1 / 3}} m, \quad \tan \beta=1,
$$

for $\kappa+\lambda<0$. We thus see that the spontaneous breaking of the global $S U(3)_{2}$ symmetry is induced by the tadpole term. To ensure that this occurs not too far above the electroweak scale, while $m$ can be in the multi-TeV range, some tuning between $\kappa$ and $\lambda$ is needed. If one quantifies the level of tuning by the parameter $\epsilon>0$, where $\kappa=-\lambda(1+\epsilon)$, then $f \simeq \mp \sqrt{\epsilon} m / \lambda^{2 / 3}$. Also,

$$
\frac{f}{v_{N}}= \pm \sqrt{2 \epsilon}
$$

implying $v_{N}>f$.

The problem of the runaway in the $S_{u}$ direction remains to be discussed. As we show now, this direction is uplifted by radiative corrections. The dominant contribution to the ColemanWeinberg one-loop effective potential for $S_{u}$,

$$
\Delta V_{1-\text { loop }}\left(S_{u}\right)=\frac{1}{64 \pi^{2}} \operatorname{STr}\left[\mathcal{M}^{4}\left(S_{u}\right)\left(\ln \frac{\mathcal{M}^{2}\left(S_{u}\right)}{\Lambda^{2}}-\frac{3}{2}\right)\right],
$$

comes from the (s)top sector. The fermion mass matrix is given by:

$$
\left(\begin{array}{ll}
t & T
\end{array}\right)\left(\begin{array}{cc}
0 & 0 \\
y_{2} S_{u} & y_{1} F
\end{array}\right)\left(\begin{array}{c}
t^{c} \\
T^{c}
\end{array}\right)
$$

(where we have frozen $\left\langle H_{u}\right\rangle=0$ ), and has a single nonzero eigenvalue corresponding to the mass of the heavy top quark, $m_{T}=\sqrt{\left|y_{2} S_{u}\right|^{2}+\left|y_{1} F\right|^{2}}$. Neglecting the small difference between the soft masses of the stop fields (see Appendix B), the (s)top sector contribution to the one-loop effective potential reads:

$$
\Delta V_{1-\text { loop }}=\frac{3}{16 \pi^{2}}\left[\left(m_{T}^{2}+m_{\text {stop }}^{2}\right)^{2}\left(\ln \left(\frac{m_{T}^{2}+m_{\text {stop }}^{2}}{\Lambda^{2}}\right)-\frac{3}{2}\right)-m_{T}^{4}\left(\ln \left(\frac{m_{T}^{2}}{\Lambda^{2}}\right)-\frac{3}{2}\right)\right],
$$

where $m_{\text {stop }}^{2} \equiv m_{\Psi_{Q}}^{2}=m_{T^{c}}^{2}=m_{t^{c}}^{2}$. For large $S_{u}$ values, $\Delta V_{1-\text { loop }}\left(S_{u}\right)$ grows as $\left|S_{u}\right|^{2} \ln \left|S_{u}\right|^{2}$, thus curing the runaway behavior due to the $m_{u}^{2}\left|S_{u}\right|^{2}$ term in the tree-level potential. However, an unwanted minimum appears along the $S_{u}$ direction at the location:

$$
S_{u, \min }^{2} \simeq \frac{F^{2}}{y_{2}^{2}} \exp \left(-\frac{8 \pi^{2} m_{u}^{2}}{3 y_{2}^{2} m_{\mathrm{stop}}^{2}}\right), \quad N \simeq S_{d} \simeq 0
$$

where we have set $\Lambda=F$ and, consistently, $m_{u}^{2}$ stands for the running mass squared $m_{u}^{2}(F)$. The value of $S_{u, \text { min }}$ grows exponentially as the absolute value of $m_{u}^{2}$ increases. The value of the scalar potential at this minimum thus decreases exponentially with $\left|m_{u}^{2}\right|$ :

$$
V_{\text {run }}\left(S_{u, \min }\right) \simeq-\frac{3 F^{2} m_{\text {stop }}^{2}}{8 \pi^{2}} \exp \left(-\frac{8 \pi^{2} m_{u}^{2}}{3 y_{2}^{2} m_{\text {stop }}^{2}}\right),
$$

where $V_{\text {run }}\left(S_{u}\right)=m_{u}^{2}\left|S_{u}\right|^{2}+\Delta V_{1-\text { loop }}\left(S_{u}\right)$. In order to ensure that the global minimum of the scalar potential is the one approximated by Eq. (21), one has to check that $V_{\min }\left(f, v_{N}\right)<$ $V_{\text {run }}\left(S_{u, \min }\right)$, where $V_{\min }\left(f, v_{N}\right)$ is the value of the scalar potential at the minimum (21):

$$
V_{\min }\left(f, v_{N}\right) \simeq \frac{3}{2} m^{3} v_{N} \simeq-\frac{3 m^{4}}{2|2 \lambda(2 \kappa+\lambda)|^{1 / 3}} .
$$


This requirement imposes some restrictions on the parameters of the model, most notably on the messenger mass $M$ and on the tadpole scale $m$ (or equivalently on $M$ and on the parameter $\xi)$. In particular, larger values of $m$ are preferred, making it necessary to slightly tune the values of $\kappa$ and $\lambda$ in order to maintain the $S U(3)_{2}$ breaking scale $f$ below $1 \mathrm{TeV}$ or so. Numerical calculations show that $M \lesssim 1000 \mathrm{TeV}$ and $\xi \gtrsim 10^{-4}$ lead to reasonable results.

\subsection{Global $S U(3)_{2}$ symmetry breaking: numerical results}

In order to study numerically the spontaneous breaking of the global $S U(3)_{2}$ symmetry, we first set the values of the parameters $F, y_{1}, y_{2}, \kappa, \lambda$ and of the various soft terms at the messenger scale and perform the appropriate renormalization group (RG) running. The $S U(3)_{W}$ breaking scale $F$ and the coupling $y_{1}$ cannot be chosen too large if one wants to uphold the pseudoGoldstone nature of the Higgs doublet, since the $S U(3)_{2}$ violating effects in the (s)top sector are proportional to $y_{1} F$, as discussed in the next section. All the effects neglected in the analytical discussion above (like the superpotential tadpole term, the soft terms in the Higgs potential and the contributions from the Higgs sector to the Coleman-Weinberg effective potential) are taken into account numerically. The $S U(3)_{2}$-symmetric RGEs valid between the messenger scale $M$ and the $S U(3)_{W}$ breaking scale $F$ can be found in Appendix $\mathrm{A}$, while Appendix $\mathrm{B}$ gives the boundary conditions for the soft terms at the messenger scale, calculated using the wave-function renormalization technique of Ref. [13].

The minimization of the scalar potential at the scale $F$ leads to the spontaneous breaking of the $S U(3)_{W} \times U(1)_{X}$ gauge symmetry, together with the $S U(3)_{1}$ global symmetry. Then the heavy degrees of freedom are integrated out and the breaking of the global $S U(3)_{2}$ symmetry is studied by minimizing the effective potential below $F$, defined as the sum of the tree-level potential (14) with its parameters renormalized at the scale $F$ and of the Coleman-Weinberg one-loop corrections computed with $\Lambda=F$. The result is then confronted with the requirement of having the proper global minimum of the potential, with the $S U(3)_{2}$ breaking scale $f$ not too far above the electroweak scale, and a correct prediction for the top quark Yukawa coupling. The approximate proportionality between the VEVs $v_{N}, f$ and the tadpole scale $m$, Eq. (21), is confirmed by numerical calculations. A slightly more accurate set of equations for $v_{N}$ and $f$ is given by:

$$
\begin{gathered}
f^{2} \simeq \frac{1}{\lambda^{2}}\left[-2 \lambda(\kappa+\lambda) v_{N}^{2}-m_{u}^{2}-m_{d}^{2}\right], \\
2 \lambda^{2}(2 \kappa+\lambda) v_{N}^{3}+(\kappa+\lambda)\left(m_{u}^{2}+m_{d}^{2}\right) v_{N}-\lambda m^{3} \simeq 0,
\end{gathered}
$$

and the prediction $\tan \beta \simeq 1$ holds. Figs. 1 and 2 illustrate the dependence of the $S U(3)_{2}$ breaking scale $f$ on the parameters $\kappa$ and $\lambda$, for a messenger mass $M=500 \mathrm{TeV}$, a typical soft mass scale $m_{\text {soft }} \sim 1 \mathrm{TeV}$ and various choices for $\xi, y_{1}$ and $F$. All coupling values in the figures are given at the messenger scale. As shown by these plots, $f \lesssim 1 \mathrm{TeV}$ can be achieved with $\lambda \sim 1$ and a mild tuning between $\kappa$ and $\lambda, \epsilon \sim 0.2$. These values imply a moderate hierarchy between the $S U(3)_{2}$ breaking scale $f$ and the VEV $v_{N}$.

\subsection{The solution of the $\mu / B \mu$ problem}

Let us now discuss the generation of the $\mu$ and $B \mu$ terms. After spontaneous breaking of the global $S U(3)_{2}$ symmetry, the doublet and singlet components of the Higgs triplets $\mathcal{H}_{u}$ and $\mathcal{H}_{d}$ no longer share the same masses and couplings, and the quadratic part of the tree-level scalar 


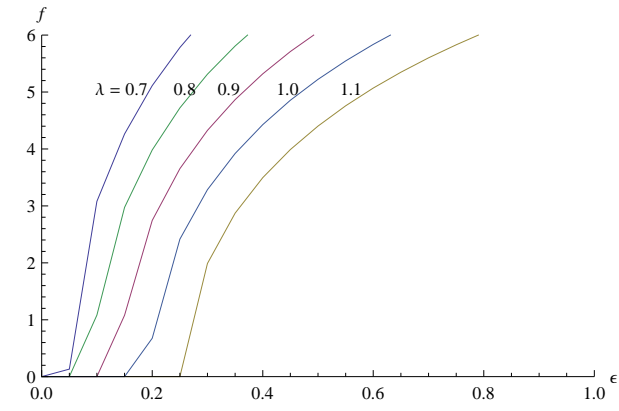

Figure 1: $f[\mathrm{TeV}]$ as a function of $\lambda$ and $\epsilon$, for $M=500 \mathrm{TeV}, m_{\mathrm{soft}} \sim 1 \mathrm{TeV}, y_{1}=0.1$, $F=10 \mathrm{TeV}$ and $\xi=0.002$.

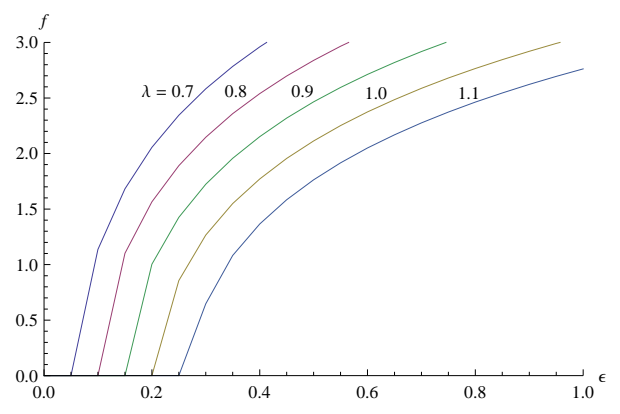

Figure 2: $f[\mathrm{TeV}]$ as a function of $\lambda$ and $\epsilon$, for $M=500 \mathrm{TeV}, m_{\mathrm{soft}} \sim 1 \mathrm{TeV}, y_{1}=1$, $F=7 \mathrm{TeV}$ and $\xi=0.00014$.

potential (14) can be rewritten as:

$$
\begin{aligned}
V_{\text {quadr. }}= & |\mu|^{2}\left(\left|H_{u}\right|^{2}+\left|H_{d}\right|^{2}\right)+\left(B \mu H_{u} \cdot H_{d}+\text { h.c. }\right) \\
& +\frac{1}{2}\left(\begin{array}{lll}
s_{u} & s_{d} & s_{N}
\end{array}\right) M_{S}^{2}\left(\begin{array}{c}
s_{u} \\
s_{d} \\
s_{N}
\end{array}\right)+\frac{1}{2}\left(p_{u} p_{d} p_{N}\right) M_{P}^{2}\left(\begin{array}{c}
p_{u} \\
p_{d} \\
p_{N}
\end{array}\right),
\end{aligned}
$$

where $H_{u} \cdot H_{d} \equiv H_{u}^{T} i \sigma^{2} H_{d}, S_{u, d} \equiv f_{u, d}+\left(s_{u, d}+i p_{u, d}\right) / \sqrt{2}, N \equiv v_{N}+\left(s_{N}+i p_{N}\right) / \sqrt{2}$ and all parameters in the scalar potential are assumed to be real. As in the NMSSM, the $\mu$ and $B \mu$ parameters are generated by the VEVs of the scalar and F-term components of the singlet superfield $N$ (the signs in the expressions for $\mu$ and $B \mu$ are due to the fact that $\mathcal{H}_{u} \mathcal{H}_{d}=$ $\left.-H_{u}^{T} i \sigma^{2} H_{d}+S_{u} S_{d}\right)$ :

$$
\mu=-\lambda v_{N}, \quad B \mu=\lambda F_{N}-\lambda A_{\lambda} v_{N}=-\lambda\left(\lambda f_{u} f_{d}+\kappa v_{N}^{2}+A_{\lambda} v_{N}\right) .
$$

However, there is a crucial difference with the NMSSM: here $F_{N}$ receives a contribution from the VEVs of $S_{u}$ and $S_{d}$, which transform non-trivially under the global $S U(3)_{2}$ symmetry. At the minimum of the tree-level scalar potential (14), these VEVs take values such that the relation $(B \mu)^{2}=\left(\mu^{2}+m_{u}^{2}\right)\left(\mu^{2}+m_{d}^{2}\right)$ is satisfied. This in turns implies that the determinant of the $\left(H_{u}\right.$, $H_{d}$ ) mass matrix vanishes (a similar mechanism is at work in the $S U(3)$-symmetric version of the model of Ref. [2]). The massless combination:

$$
H=\sin \beta\left(i \sigma^{2} H_{u}^{*}\right)+\cos \beta H_{d},
$$

to be identified with the Standard Model Higgs boson, is interpreted as a Goldstone boson of the spontaneously broken global $S U(3)_{2}$ symmetry. The orthogonal combination $H^{\prime}$ is heavy with a mass $m_{H^{\prime}}^{2}=2 \mu^{2}+m_{u}^{2}+m_{d}^{2}$. Inspection of the singlet scalar and pseudoscalar mass matrices $M_{S}^{2}$ and $M_{P}^{2}$ show that there is another Goldstone boson,

$$
\eta=\sin \beta p_{u}-\cos \beta p_{d} .
$$

The other singlets are massive with masses of order a few $\mu$, except for a lighter one with a mass of order $\lambda f \lesssim 1 \mathrm{TeV}$.

If we restrict our attention to the part of the tree-level scalar potential that depends solely on $H$, we see no dependence on $\mu$ and $B \mu$, while the masses of the heavy states of the Higgs sector (including the higgsinos) are of order $\mu$. Hence, the electroweak scale is insensitive to the actual value of the $\mu$ parameter, which is allowed to be large without creating a strong fine-tuning in 
the Higgs potentia $\sqrt{5}$. This elegantly solves the $\mu / B \mu$ problem of gauge mediation. The value of $\mu$ (or $v_{N}$ ) is relevant, on the other hand, for the breaking of the global symmetry, and we have seen in the previous subsections that a moderate hierarchy $\mu \gg f$ is needed, with no incidence on fine-tuning by virtue of the global $S U(3)_{2}$ symmetry.

\section{Electroweak symmetry breaking}

We are now in a position to discuss the breaking of the electroweak symmetry. Let us first recapitulate the identification of the light degrees of freedom in the Higgs sector. The spontaneous breaking of the global $S U(3)_{1} \times S U(3)_{2}$ symmetry leads to 10 Goldstone bosons, 5 of which disappear from the massless spectrum by virtue of the Higgs mechanism, since the gauge symmetry $S U(3)_{W} \times U(1)_{X}$ is broken to $S U(2)_{L} \times U(1)_{Y}$ in the same process. The remaining 5 Goldstone bosons reside mainly in $\mathcal{H}_{u}$ and $\mathcal{H}_{d}$ in the limit $f \ll F$, and are conveniently parameterized a. 6 [5]:

$$
\mathcal{H}_{d}=f_{d}\left(\begin{array}{c}
\frac{H}{|H|} \sin \left(\frac{|H|}{f}\right) \\
e^{-\frac{i \eta}{f \sqrt{2}} \cos \left(\frac{|H|}{f}\right)}
\end{array}\right), \quad \mathcal{H}_{u}=f_{u}\left(\frac{H^{\dagger}}{|H|} \sin \left(\frac{|H|}{f}\right), \quad e^{\frac{i \eta}{f \sqrt{2}}} \cos \left(\frac{|H|}{f}\right)\right),
$$

where $f_{u} \equiv f \sin \beta, f_{d} \equiv f \cos \beta$ and $|H| \equiv \sqrt{H^{\dagger} H}$. All other components of the Higgs triplets, except for one singlet with mass of order $\lambda f$, are heavy with masses of order a few $\mu \gg f$ and can be integrated out. $H$ is a Standard Model-like Higgs doublet transforming as a $\mathbf{2}_{-1 / 2}$ of $S U(2)_{L} \times U(1)_{Y}$, while $\eta$ is a singlet whose role has been discussed in Refs. [5, 11, 12]. Being a (pseudo-)Goldstone boson of the approximate global $S U(3)_{2}$ symmetry, $H$ gets its potential from $S U(3)_{2}$ breaking interactions. At tree level, one has:

$$
V_{\text {tree }}(H)=V_{\text {light }}(H)+V_{\text {heavy }}(H),
$$

where $V_{\text {light }}(H)$ is the contribution of the $S U(2)_{L} \times U(1)_{Y} D$-terms:

$$
V_{\text {light }}(H)=\lambda_{0}\left\{|H|^{4}+\mathcal{O}\left(\frac{|H|^{6}}{f^{2}}\right)\right\}, \quad \lambda_{0}=\frac{g^{2}+g^{\prime 2}}{8} \cos ^{2} 2 \beta,
$$

and $V_{\text {heavy }}(H)$ is the contribution of the terms $\delta V_{\text {soft }}$ left over by integrating out the heavy gauge supermultiplets at the scale $F$ :

$$
V_{\text {heavy }}(H)=m_{0}^{2}\left\{|H|^{2}+\mathcal{O}\left(\frac{|H|^{4}}{f^{2}}\right)\right\}, \quad m_{0}^{2}=\frac{9-21 t_{W}^{2}+4 t_{W}^{4}}{36}\left(m_{D}^{2}-m_{U}^{2}\right)(-\cos 2 \beta),
$$

$\left(m_{0}^{2}>0\right.$ due to $\cos 2 \beta<0$ and $\left.m_{D}^{2}-m_{U}^{2}>0\right)$. Since $\tan \beta \simeq 1$, both the tree-level quartic coupling $\lambda_{0}$ and the mass parameter $m_{0}^{2}$ are smal 7 . As we are going to see in the next subsection, one-loop corrections induced by the large top quark Yukawa coupling generate a tachyonic mass term in the Higgs potential and trigger electroweak symmetry breaking. The electroweak scale $v$ is related to the VEV of the Higgs doublet $\bar{v} \equiv\langle H\rangle$ by:

$$
v=f \sin (\bar{v} / f) .
$$

\footnotetext{
${ }^{5}$ In fact, radiative corrections induce a dependence of the one-loop effective potential on $\mu$ and $B \mu$ (see Section 4.1), but this does not represent an important source of fine-tuning.

${ }^{6}$ This parametrization agrees with Eqs. (33) and (34) at leading order in $1 / f$.

${ }^{7}$ As discussed at the beginning of Section $3 m_{0}^{2}$ is further suppressed by the small RG-induced difference of heavy Higgs triplet soft masses $m_{D}^{2}-m_{U}^{2}$. It is therefore not expected to play a significant role in the dynamics of electroweak symmetry breaking. This is confirmed by numerical calculations, which show that the tree-level contributions to the Higgs potential are negligible in comparison with the one-loop corrections.
} 


\subsection{Electroweak symmetry breaking: analytical discussion}

At the one-loop level, $V(H)$ receives contributions from the Higgs couplings to the matter and gauge fields, which explicitly break the global $S U(3)_{2}$ symmetry. Let us first compute the radiative corrections induced by the top quark Yukawa coupling, using the Coleman-Weinberg formula (Eq. (23) with $S_{u}$ replaced by $H$ ). The fermion mass matrix squared reads, in the parameterization (35):

$$
M_{\text {top }}^{\dagger} M_{\text {top }}=\left(\begin{array}{cc}
y_{2}^{2} f_{u}^{2} & y_{1} y_{2} F f_{u} \cos (|H| / f) \\
y_{1} y_{2} F f_{u} \cos (|H| / f) & y_{1}^{2} F^{2}
\end{array}\right),
$$

where $\mathcal{L}_{\text {mass }} \ni-(t T) M_{\text {top }}\left(t^{c} T^{c}\right)^{T}+$ h.c. . The eigenstates can be identified with the Standard Model top quark and its heavy $S U(3)$ partner, with masses:

$$
\left(m_{t}^{T}\right)^{2}=\frac{1}{2}\left(y_{1}^{2} F^{2}+y_{2}^{2} f_{u}^{2} \pm \sqrt{\left(y_{1}^{2} F^{2}+y_{2}^{2} f_{u}^{2}\right)^{2}-4 y_{1}^{2} y_{2}^{2} F^{2} f_{u}^{2} \sin ^{2}(|H| / f)}\right) .
$$

For $|H| \ll f$, Eq. (41) simplifies to:

$$
m_{t}^{2} \simeq y_{t}^{2}|H|^{2}, \quad m_{T}^{2} \simeq y_{1}^{2} F^{2}+y_{2}^{2} f_{u}^{2},
$$

where

$$
y_{t}^{2}=\frac{y_{1}^{2} y_{2}^{2} F^{2} \sin ^{2} \beta}{y_{1}^{2} F^{2}+y_{2}^{2} f_{u}^{2}} .
$$

Plugging these expressions into the Coleman-Weinberg formula and neglecting the small difference between the soft masses of the stop fields, one obtains similar expressions to the ones of Ref. [5]:

$$
\delta_{t} m_{H}^{2} \simeq-\frac{3 y_{t}^{2}}{8 \pi^{2}}\left[m_{\text {stop }}^{2} \ln \left(1+\frac{m_{T}^{2}}{m_{\text {stop }}^{2}}\right)+m_{T}^{2} \ln \left(1+\frac{m_{\text {stop }}^{2}}{m_{T}^{2}}\right)\right]
$$

and

$$
\begin{aligned}
\delta_{t} \lambda_{H} \simeq \frac{3 y_{t}^{4}}{16 \pi^{2}} & {\left[\ln \left(\frac{m_{\text {stop }}^{2} m_{T}^{2}}{m_{t}^{2}\left(m_{\text {stop }}^{2}+m_{T}^{2}\right)}\right)-2 \frac{m_{\text {stop }}^{2}}{m_{T}^{2}} \ln \left(1+\frac{m_{T}^{2}}{m_{\text {stop }}^{2}}\right)\right.} \\
+ & \left.\frac{2 m_{\text {stop }}^{2}}{3 y_{t}^{2} f^{2}} \ln \left(\frac{m_{\text {stop }}^{2}+m_{T}^{2}}{m_{\text {stop }}^{2}}\right)+\frac{2 m_{T}^{2}}{3 y_{t}^{2} f^{2}} \ln \left(\frac{m_{\text {stop }}^{2}+m_{T}^{2}}{m_{T}^{2}}\right)\right],
\end{aligned}
$$

where $\delta_{t} m_{H}^{2}$ and $\delta_{t} \lambda_{H}$ are the contributions of the (s)top sector to the coefficients of the quadratic and quartic terms in the one-loop effective Higgs potential, respectively:

$$
\Delta V_{1-\text { loop }}(H)=\delta m_{H}^{2}|H|^{2}+\delta \lambda_{H}|H|^{4}+\cdots .
$$

As required for proper electroweak symmetry breaking, $\delta_{t} m_{H}^{2}$ is negative while $\delta_{t} \lambda_{H}$ is positive. The absence of a $\ln \Lambda$-dependent piece in $\delta_{t} m_{H}^{2}$ is a direct consequence of the double protection of the Higgs mass by supersymmetry and by the global $S U(3)_{2}$ symmetry.

Let us now consider the contributions of the gauge interactions to $\delta m_{H}^{2}$ and $\delta \lambda_{H}$. In the effective theory below the gauge symmetry breaking scale $F$, these depend logarithmically on the cut-off scale $\Lambda$ due to the explicit breaking of the global $S U(3)_{2}$ symmetry by the $S U(2)_{L} \times U(1)_{Y}$ gauge couplings and gaugino masses. The dominant contributions to the one-loop effective potential are given by the approximate formula:

$$
\Delta_{\text {gauge }} V_{1-\text { loop }}(H) \simeq-\frac{1}{64 \pi^{2}} \operatorname{STr}\left[\mathcal{M}_{\text {gauge-Higgs }}^{4}(H) \ln \frac{\Lambda^{2}}{m_{\text {soft }}^{2}}\right],
$$


where $\mathcal{M}_{\text {gauge-Higgs }}^{4}$ stands for the (fourth power of the) mass matrices of the gauge and Higgs fields, and $m_{\text {soft }}$ is an average soft mass. Since the whole gauge sector is $S U(3)_{2}$ symmetric above the scale $F$, the logarithmic divergence is effectively cut off at $\Lambda=F$. Let us compute (47). Working in the approximation where the heavy gauge and Higgs fields are integrated out at tree level, one is left with the $S U(2)_{L} \times U(1)_{Y}$ gauge fields and with the Higgs superfields $\mathcal{H}_{u}, \mathcal{H}_{d}$ and $N$. Using the Higgs superpotential $W=\lambda N \mathcal{H}_{u} \mathcal{H}_{d}+\frac{\kappa}{3} N^{3}$, the tree-level potential (14)(19) and the Lagrangian terms involving the $S U(2)_{L} \times U(1)_{Y}$ gauge fields, one derives the mass matrices of the gauge bosons, charginos, neutralinos, charged and neutral Higgs bosons. Neglecting $\delta V_{\text {soft }}$ as well as the soft terms that are suppressed by the small parameter $\xi$, and assuming all parameters to be real, one obtains:

$$
\begin{gathered}
\operatorname{STr}\left[\mathcal{M}_{\text {gauge-Higgs }}^{4}\right]=-\left[4\left(3 g^{2} M_{2}^{2}+g^{\prime 2} M_{1}^{2}\right)+3\left(3 g^{2}+g^{\prime 2}\right) \mu^{2}\right]\left(\left|H_{u}\right|^{2}+\left|H_{d}\right|^{2}\right) \\
+\left(\left[4\left(3 g^{2} M_{2}+g^{\prime 2} M_{1}\right) \mu-\left(3 g^{2}+g^{\prime 2}\right) B \mu\right] H_{u} \cdot H_{d}+\text { h.c. }\right) \\
+3\left(g^{2}+g^{\prime 2}\right)\left[m_{u}^{2}\left|H_{u}\right|^{2}+m_{d}^{2}\left|H_{d}\right|^{2}\right]-2 g^{\prime 2}\left[m_{d}^{2}\left|H_{u}\right|^{2}+m_{u}^{2}\left|H_{d}\right|^{2}\right] \\
+\left[\frac{\left(g^{2}+g^{\prime 2}\right)^{2}}{2}-\frac{9}{4} g^{4}+\frac{1}{4} g^{\prime 4}-\lambda^{2}\left(g^{2}+g^{\prime 2}\right)\right]\left(\left|H_{u}\right|^{2}-\left|H_{d}\right|^{2}\right)^{2}
\end{gathered}
$$

where field-independent terms have been omitted, and $\mu=-\lambda v_{N}, B \mu=-\lambda\left(\lambda f_{u} f_{d}+\kappa v_{N}^{2}\right)$. Inserting the parameterization (35) into Eq. (48), one finally obtains:

$$
\begin{aligned}
\delta_{g} m_{H}^{2} \simeq & \left\{\frac{3 g^{2} M_{2}^{2}+g^{\prime 2} M_{1}^{2}}{16 \pi^{2}}+\frac{3}{64 \pi^{2}}\left(3 g^{2}+g^{\prime 2}\right) \mu^{2}+\frac{3 g^{2} M_{2}+g^{\prime 2} M_{1}}{16 \pi^{2}} \mu \sin 2 \beta\right. \\
& -\frac{3 g^{2}+g^{\prime 2}}{64 \pi^{2}} B \mu \sin 2 \beta-\frac{3\left(g^{2}+g^{\prime 2}\right)}{64 \pi^{2}}\left[m_{u}^{2} \sin ^{2} \beta+m_{d}^{2} \cos ^{2} \beta\right] \\
& \left.+\frac{g^{\prime 2}}{32 \pi^{2}}\left[m_{d}^{2} \sin ^{2} \beta+m_{u}^{2} \cos ^{2} \beta\right]\right\} \ln \left(\frac{F^{2}}{m_{\text {soft }}^{2}}\right), \\
\delta_{g} \lambda_{H} \simeq & -\frac{1}{64 \pi^{2}}\left[\frac{\left(g^{2}+g^{\prime 2}\right)^{2}}{2}-\frac{9}{4} g^{4}+\frac{1}{4} g^{\prime 4}-\lambda^{2}\left(g^{2}+g^{\prime 2}\right)\right] \cos ^{2} 2 \beta \ln \left(\frac{F^{2}}{m_{\text {soft }}^{2}}\right) \\
& -\frac{\delta_{g} m_{H}^{2}}{3 f^{2}},
\end{aligned}
$$

where we have set $\Lambda=F$. The terms enhanced by $\mu^{2}$ and $\mu M_{1,2}$ dominate in $\delta_{g} m_{H}^{2}$, so that $\delta_{g} m_{H}^{2}>0$. Note that there is a partial cancellation between the second and the fourth terms, due to $B \mu \simeq-\lambda \kappa v_{N}^{2} \simeq \lambda^{2} v_{N}^{2}=\mu^{2}$, leaving a net contribution $\left(3 g^{2}+g^{\prime 2}\right) \mu^{2} \ln \left(F^{2} / m_{\text {soft }}^{2}\right) / 32 \pi^{2}$. Contrary to $\delta_{t} \lambda_{H}, \delta_{g} \lambda_{H}$ is negative, but it is suppressed by $\cos ^{2} 2 \beta$ (first term) and by $1 / 3 f^{2}$ (second term).

The second and fourth terms in Eq. (49), which due to the large value of $\mu$ (see next subsection) give the dominant contribution from the gauge-Higgs sector to the Coleman-Weinberg potential, have a simple renormalization group interpretation. They arise from the different RG running, below the $S U(3)_{W}$ breaking scale $F$, of the parameters associated with the doublet and singlet components of the Higgs triplets $\mathcal{H}_{u}$ and $\mathcal{H}_{d}$. Indeed, below $F$, gauge interactions distinguish the doublets $H_{u}$ and $H_{d}$ from their $S U(3)$ partners $S_{u}$ and $S_{d}$, and this results in different RGEs for parameters that would otherwise be equal by virtue of the $S U(3)_{2}$ symmetry. One is thus led to "split" the superpotential coupling $\lambda$ in the following way:

$$
W_{\text {Higgs }} \ni \lambda_{s} N S_{u} S_{d}+\lambda_{d} N H_{u} H_{d}
$$

and similarly for the soft terms involving $\mathcal{H}_{u}$ or $\mathcal{H}_{d}$. As a result, the $F$-term potential (15) is modified as follows:

$$
V_{F}=\left(\left[\lambda_{s} \cos ^{2}\left(\frac{|H|}{f}\right)+\lambda_{d} \sin ^{2}\left(\frac{|H|}{f}\right)\right] f^{2} \sin \beta \cos \beta+\kappa v_{N}^{2}\right)^{2}
$$




$$
+v_{N}^{2} f^{2}\left(\lambda_{s}^{2} \cos ^{2}\left(\frac{|H|}{f}\right)+\lambda_{d}^{2} \sin ^{2}\left(\frac{|H|}{f}\right)\right)
$$

where we have replaced $N$ by its VEV and inserted the parameterization (35). Using $B \mu \simeq \mu^{2}$ and $\sin 2 \beta \simeq 1$, this yields:

$$
\delta_{\text {split }} m_{H}^{2} \simeq \frac{\lambda_{d}-\lambda_{s}}{\lambda} \mu^{2}, \quad \delta_{\text {split }} \lambda_{H} \simeq-\frac{\delta_{\text {split }} m_{H}^{2}}{3 f^{2}},
$$

where $\lambda_{d}-\lambda_{s} / \lambda$ can be computed from the RGEs for the "split" superpotential couplings given in Appendix C:

$$
\frac{\lambda_{d}-\lambda_{s}}{\lambda} \simeq \ln \frac{\lambda_{d}}{\lambda_{s}} \simeq \frac{3 g^{2}+g^{2}}{32 \pi^{2}} \ln \left(\frac{F^{2}}{m_{\mathrm{soft}}^{2}}\right)
$$

in agreement with the second and fourth terms of Eq. (49).

\subsection{Electroweak symmetry breaking: numerical results}

The numerical study of electroweak symmetry breaking is done by minimizing the Higgs potential, taking into account the contributions mentioned in the previous subsections and relaxing the assumptions made in the analytical discussion (in particular, the soft masses in the stop sector are not universal but given by the formulae of Appendix B). For simplicity, only the dominant (s)top sector contribution and the approximate gauge contribution (47)-(48) have been included in the numerical computation of the Coleman-Weiberg potential.

Let us comment on the features of the main contributions to the Higgs potential, as revealed by the numerical calculations. The contribution from the (s)top sector to the Coleman-Weinberg one-loop effective potential has the desired "mexican hat" shape with the minimum approximately located at $\bar{v} \approx \frac{\pi}{2} f$. The gauge contribution is convex for small values of the Higgs VEV with a minimum at the origin, which helps shifting the minimum of the Higgs potential towards the correct value $\bar{v} \approx v=174 \mathrm{GeV}$. However, the LEP bound on the Higgs mass requires large corrections from the (s)top sector to the quartic coupling $\lambda_{H}$ and at the same time does not allow for large corrections from the gauge-Higgs sector (which gives $\delta_{g} \lambda_{H}<0$ ). This in turn implies that the gauge contribution (47)-(48) is not enough to bring $v$ to its true value. Conversely, one may adjust the parameters of the model so that the correct value of the electroweak scale is obtained, but then the radiative corrections to $\lambda_{H}$ are too small and the Higgs mass falls below the LEP bound. This means that the model must be extended to be fully realistic. A first way to do so is to add an $S U(3)_{2}$-breaking sector that generates a sizeable tree-level quartic coupling $\lambda_{0}$, as was done in a different $S U(3)$ model in Ref. [6]. Then large corrections from the (s)top sector are no longer needed to satisfy the LEP bound, and the electroweak scale is obtained with little fine-tuning. Another possibility is to invoke some additional convex contribution $\delta_{\text {extra }} m_{H}^{2}|H|^{2}$ to the effective Higgs potential (presumably arising from loops involving the heavy gauge and Higgs fields, or from some extra $S U(3)_{2}$-breaking sector to be added to the model) in order to obtain the proper value of the Higgs VEV. In this case large corrections from the (s)top sector to $\lambda_{H}$ are still needed to satisfy the LEP constraint and the fine-tuning is more significant.

Let us investigate the second possibility. To fix the size of the (s)top and gauge contributions, we require that the resulting $\delta \lambda_{H}$ be large enough to satisfy the LEP bound for a mass of the $S U(3)$ top partner $m_{T}$ in the $1-10 \mathrm{TeV}$ range. In practice, the parameter values chosen in Fig. 2 for the spontaneous breaking of the global $S U(3)_{2}$ symmetry turn out to be convenient for that purpose and we adopt them in our numerical study. Then we adjust the extra contribution $\delta_{\text {extra }} m_{H}^{2}|H|^{2}$ to obtain the correct value of the electroweak scale. Let us now present the numerical results for the same choice of parameters as in Fig. 2 (all coupling values in the figures are given at the messenger scale). The value of the Higgs mass $M_{h} \simeq 2 \sqrt{\lambda_{0}+\delta \lambda_{H}} v$ 
is displayed in Fig. 3 (the dashed lines show the Higgs mass predicted by the (s)top sector contribution to the effective potential alone), while the corresponding value of $\tan \beta$ is shown in Fig. 4. The curves in Figs. 4 to 6 are dashed in the region of the parameter space where the Higgs mass lies below the LEP bound.

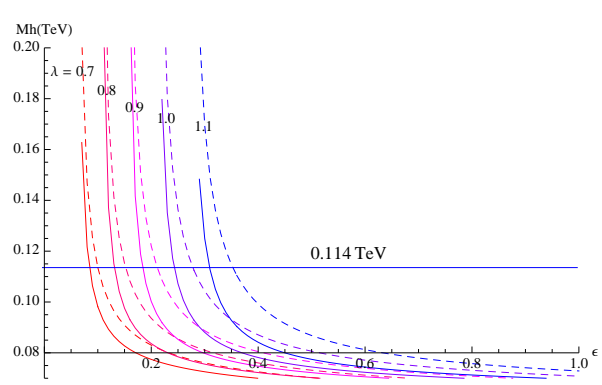

Figure 3: Higgs boson mass as a function of $\lambda$ and $\epsilon$, for $M=500 \mathrm{TeV}, m_{\text {soft }} \sim 1 \mathrm{TeV}$, $y_{1}=1, F=7 \mathrm{TeV}$ and $\xi=0.00014[\mathrm{in} \mathrm{TeV}]$.

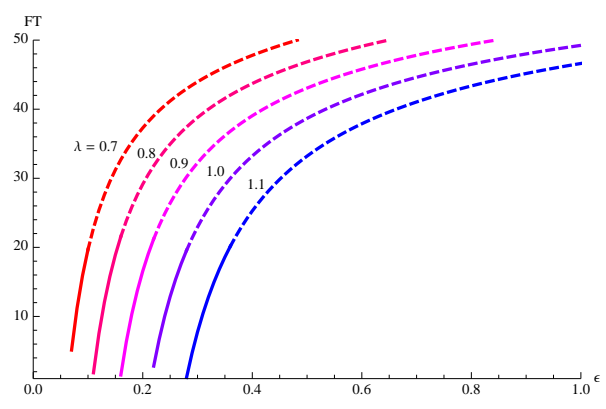

Figure 5: Fine-tuning as a function of $\lambda$ and $\epsilon$. Other parameters chosen as in Fig. 3

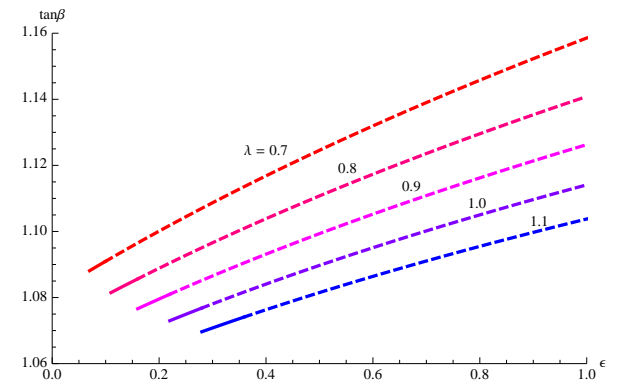

Figure 4: $\tan \beta$ as a function of $\lambda$ and $\epsilon$. Other parameters chosen as in Fig. [3.

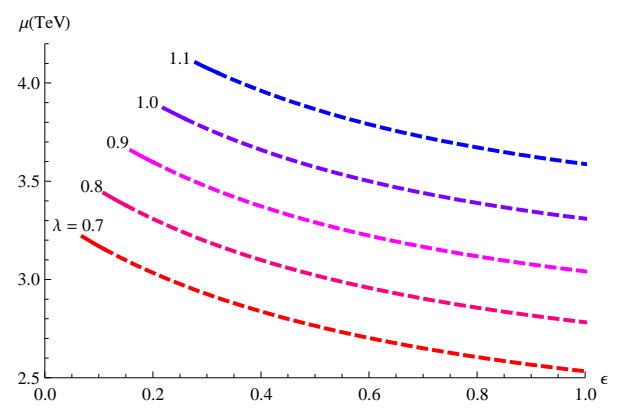

Figure 6: $\mu=-\lambda v_{N}$ as a function of $\lambda$ and $\epsilon$. Other parameters chosen as in Fig. 3.

The source of fine-tuning in the model lies in the large radiative corrections to $\lambda_{H}$ from the (s)top sector that are required in order to satisfy the LEP bound. This in turn implies a large and negative $\delta_{t} m_{H}^{2}$ that must be compensated for by $\delta_{g} m_{H}^{2}$ and $\delta_{\text {extra }} m_{H}^{2}$ so as to obtain the proper value of the electroweak scale $v=174 \mathrm{GeV}$. One can estimate this fine-tuning with the following quantity:

$$
F T=\left|\frac{\left|\delta_{t} m_{H}^{2}\right|-\left|\delta m_{H}^{2}\right|}{\delta m_{H}^{2}}\right|,
$$

where $\delta m_{H}^{2}=\delta_{t} m_{H}^{2}+\delta_{g} m_{H}^{2}+\delta_{\text {extra }} m_{H}^{2}$ is the mass squared parameter in the Higgs potential (46). The numerical results for the fine-tuning parameter $F T$ are presented in Fig. 5 . Figs. 3 to 5 show that successful electroweak symmetry breaking with a Higgs boson mass above the LEP bound and a fine-tuning around $F T \sim 20$ can be achieved for $\lambda \sim 1, \epsilon \sim 0.3$ and $\xi \sim 10^{-4}$ (corresponding to $f \sim 1 \mathrm{TeV}$ ).

\subsection{Physical spectrum}

Let us finally discuss the physical spectrum of the model. The gross features of the Higgs spectrum are the following (omitting the heavy fields $\Phi_{U}, \Phi_{D}$ and $N^{\prime}$, which have masses of order $F$, out of reach of the LHC). The spontaneous breaking of the $S U(3)_{2}$ symmetry yields a massive Higgs doublet $H^{\prime} \sim \cos \beta\left(i \sigma^{2} H_{u}^{*}\right)-\sin \beta H_{d}$, which describes a CP-even and a CP-odd neutral 
scalars as well as a charged one, all with the same tree-level mass $\sqrt{2 \mu^{2}+m_{u}^{2}+m_{d}^{2}} \approx \sqrt{2}|\mu|$, hence in the multi-TeV range (see Fig. 6). In the singlet sector, we have 4 heavy scalars with masses of order a few $\mu$ and a lighter one with a mass of order $\lambda f \lesssim 1 \mathrm{TeV}$; the remaining singlet $\eta$ is a pseudo-Goldstone boson and gets a small mass at the one-loop level [11]. Apart from this singlet, whose phenomenology has been studied in Refs. [5, 11, 12], the Higgs sector contains a single light state with Standard Model-like properties.

The higgsinos (both doublet and singlets) also have large masses of order $\mu$. The rest of the superpartner spectrum is representative of gauge-mediated models with a low messenger scale. The $S U(3)$ partner of the top quark has a mass $m_{T} \simeq y_{1} F \approx 7 \mathrm{TeV}$ in the region of parameter space considered and is not accessible at the LHC, similarly to the heavy gauge bosons associated with the broken $S U(3)_{W} \times U(1)_{X}$ generators.

\section{Conclusions}

In this paper, we studied the interplay between the spontaneous breaking of a global symmetry of the Higgs sector and gauge-mediated supersymmetry breaking, in the framework of a supersymmetric model with global $S U(3)$ symmetry. In addition to solving the supersymmetric flavour problem and alleviating the little hierarchy problem by identifying the Higgs boson with a pseudo-Goldstone boson, this scenario presents several advantages.

First, gauge mediation provides a mechanism for breaking the global symmetry protecting the Higgs mass, namely through the loop-induced tadpole of an $S U(3)$ singlet scalar field. A non-trivial success of the model studied in this paper, compared with previous attempts in the literature, is to ensure that the global symmetry breaking vacuum is indeed the global minimum of the scalar potential, and the possibility to control the shape of the potential by varying the tadpole scale is instrumental in this.

Second, the global symmetry provides an elegant solution to the $\mu / B \mu$ problem of gauge mediation. Much like in the NMSSM, the $\mu$ and $B \mu$ parameters are generated by the VEVs of the scalar and F-term components of a singlet superfield, but the global $S U(3)$ symmetry ensures that the relation $(B \mu)^{2}=\left(|\mu|^{2}+m_{u}^{2}\right)\left(|\mu|^{2}+m_{d}^{2}\right)$ is satisfied at the minimum of the tree-level scalar potential, implying that the electroweak scale is insensitive to the actual value of the $\mu$ parameter. As a result the $\mu$ parameter, which sets the scale of the heavy Higgs masses, may be large without creating a strong fine-tuning in the Higgs potential.

Finally, the combined effect of supersymmetry and of the global symmetry ensures a "double protection" of the Higgs potential, allowing for a reduced fine-tuning with respect to the MSSM. We computed the one-loop corrections to the potential of the pseudo-Goldstone Higgs boson coming from the (s)top and gauge-Higgs sectors, and checked that they indeed trigger electroweak symmetry breaking. However, the specific model studied in this paper has a suppressed tree-level quartic Higgs coupling and fails to bring the Higgs mass above the LEP bound. We showed that an additional contribution $\delta_{\text {extra }} m_{H}^{2}|H|^{2}$ to the Higgs potential, arising from some extra $S U(3)$-breaking sector, can solve this problem with a moderate fine-tuning of order 1/20. Alternatively, one may try to generate a sizeable tree-level quartic Higgs coupling along the lines of Ref. [6].

The model predicts a rather low messenger scale, a small $\tan \beta$ value, a light Higgs boson with Standard Model-like properties, and heavy higgsinos.

\section{Acknowledgments}

The work of AK was supported by the MNiSzW scientific research grant N N202 103838 (2010 2012). The work of SL was partially supported by the European Community under the contracts 
MTKD-CT-2005-029466 and PITN-GA-2009-237920.

\section{A Renormalization Group Equations}

In this appendix, we give the renormalization group equations (RGEs) valid between the messenger scale $M$ and the $S U(3)_{W}$ breaking scale $F$ for all relevant superpotential parameters and soft terms. For convenience, we recall their definition below:

$$
\begin{aligned}
W \ni & \lambda^{\prime} N^{\prime}\left(\Phi_{U} \Phi_{D}-\mu^{2}\right)+\lambda N \mathcal{H}_{u} \mathcal{H}_{d}+\frac{\kappa}{3} N^{3}+y_{1} \Phi_{U} \Psi_{Q} T^{c}+y_{2} \mathcal{H}_{u} \Psi_{Q} t^{c}, \\
V_{\text {soft }} \ni & M_{U}^{2}\left|\Phi_{U}\right|^{2}+M_{D}^{2}\left|\Phi_{D}\right|^{2}+M_{N^{\prime}}^{2}\left|N^{\prime}\right|^{2}+m_{u}^{2}\left|\mathcal{H}_{u}\right|^{2}+m_{d}^{2}\left|\mathcal{H}_{d}\right|^{2}+m_{N}^{2}|N|^{2} \\
& +m_{\Psi_{Q}}^{2}\left|\Psi_{Q}\right|^{2}+m_{t^{c}}^{2}\left|m_{t^{c}}\right|^{2}+m_{T^{c}}^{2}\left|m_{T^{c}}\right|^{2}+\left(\lambda^{\prime} A_{\lambda^{\prime}} N^{\prime} \Phi_{U} \Phi_{d}\right. \\
& \left.+\lambda A_{\lambda} N \mathcal{H}_{u} \mathcal{H}_{d}+\frac{\kappa}{3} A_{\kappa} N^{3}+y_{1} A_{y_{1}} \Phi_{U} \Psi_{Q} T^{c}+y_{2} A_{y_{2}} \mathcal{H}_{u} \Psi_{Q} t^{c}+\text { h.c. }\right) .
\end{aligned}
$$

In the RGEs below, $g_{C}, g_{W}$ and $g_{X}$ are the $S U(3)_{C}, S U(3)_{W}$ and $U(1)_{X}$ gauge couplings, respectively; $M_{C}, M_{W}$ and $M_{X}$ are the associated gaugino masses; and $t \equiv\left(1 / 16 \pi^{2}\right) \ln \mu$.

$$
\begin{aligned}
& \frac{d}{d t} \lambda^{\prime}=\lambda^{\prime}\left(5 \lambda^{\prime 2}+3 y_{1}^{2}-\frac{16}{3} g_{W}^{2}-\frac{4}{9} g_{X}^{2}\right), \\
& \frac{d}{d t} \lambda=\lambda\left(5 \lambda^{2}+2 \kappa^{2}+3 y_{2}^{2}-\frac{16}{3} g_{W}^{2}-\frac{4}{9} g_{X}^{2}\right), \\
& \frac{d}{d t} \kappa=3 \kappa\left(3 \lambda^{2}+2 \kappa^{2}\right) \text {, } \\
& \frac{d}{d t} y_{1}=y_{1}\left(7 y_{1}^{2}+y_{2}^{2}+\lambda^{\prime 2}-\frac{16}{3} g_{C}^{2}-\frac{16}{3} g_{W}^{2}-\frac{4}{3} g_{X}^{2}\right) \text {, } \\
& \frac{d}{d t} y_{2}=y_{2}\left(y_{1}^{2}+7 y_{2}^{2}+\lambda^{2}-\frac{16}{3} g_{C}^{2}-\frac{16}{3} g_{W}^{2}-\frac{4}{3} g_{X}^{2}\right), \\
& \frac{d}{d t} A_{\lambda^{\prime}}=10 \lambda^{\prime 2} A_{\lambda^{\prime}}+6 y_{1}^{2} A_{y_{1}}-\frac{32}{3} g_{W}^{2} M_{W}-\frac{8}{9} g_{X}^{2} M_{X}, \\
& \frac{d}{d t} A_{\lambda}=10 \lambda^{2} A_{\lambda}+4 \kappa^{2} A_{\kappa}+6 y_{2}^{2} A_{y_{2}}-\frac{32}{3} g_{W}^{2} M_{W}-\frac{8}{9} g_{X}^{2} M_{X}, \\
& \frac{d}{d t} A_{\kappa}=6\left(3 \lambda^{2} A_{\lambda}+2 \kappa^{2} A_{\kappa}\right) \text {, } \\
& \frac{d}{d t} A_{y_{1}}=14 y_{1}^{2} A_{y_{1}}+2 \lambda^{\prime 2} A_{\lambda^{\prime}}-\frac{32}{3} g_{C}^{2} M_{C}-\frac{32}{3} g_{W}^{2} M_{W}-\frac{8}{3} g_{X}^{2} M_{X} \text {, } \\
& \frac{d}{d t} A_{y_{2}}=14 y_{2}^{2} A_{y_{2}}+2 \lambda^{2} A_{\lambda}-\frac{32}{3} g_{C}^{2} M_{C}-\frac{32}{3} g_{W}^{2} M_{W}-\frac{8}{3} g_{X}^{2} M_{X} \text {, } \\
& \frac{d}{d t} m_{U}^{2}=6 y_{1}^{2}\left(m_{U}^{2}+m_{\Psi_{Q}}^{2}+m_{T^{c}}^{2}+A_{y_{1}}^{2}\right)+2 \lambda^{\prime 2}\left(m_{U}^{2}+m_{D}^{2}+m_{N^{\prime}}^{2}+A_{\lambda^{\prime}}^{2}\right) \\
& -\frac{32}{3} g_{W}^{2} M_{W}^{2}-\frac{8}{9} g_{X}^{2} M_{X}^{2} \text {, } \\
& \frac{d}{d t} m_{D}^{2}=2 \lambda^{\prime 2}\left(m_{U}^{2}+m_{D}^{2}+m_{N^{\prime}}^{2}+A_{\lambda^{\prime}}^{2}\right)-\frac{32}{3} g_{W}^{2} M_{W}^{2}-\frac{8}{9} g_{X}^{2} M_{X}^{2}, \\
& \frac{d}{d t} m_{N^{\prime}}^{2}=6 \lambda^{\prime 2}\left(m_{U}^{2}+m_{D}^{2}+m_{N^{\prime}}^{2}+A_{\lambda^{\prime}}^{2}\right) \text {, } \\
& \frac{d}{d t} m_{u}^{2}=6 y_{2}^{2}\left(m_{u}^{2}+m_{\Psi_{Q}}^{2}+m_{t^{c}}^{2}+A_{y_{2}}^{2}\right)+2 \lambda^{2}\left(m_{u}^{2}+m_{d}^{2}+m_{N}^{2}+A_{\lambda}^{2}\right) \\
& -\frac{32}{3} g_{W}^{2} M_{W}^{2}-\frac{8}{9} g_{X}^{2} M_{X}^{2} \text {, }
\end{aligned}
$$




$$
\begin{aligned}
\frac{d}{d t} m_{d}^{2}= & 2 \lambda^{2}\left(m_{u}^{2}+m_{d}^{2}+m_{N}^{2}+A_{\lambda}^{2}\right)-\frac{32}{3} g_{W}^{2} M_{W}^{2}-\frac{8}{9} g_{X}^{2} M_{X}^{2} \\
\frac{d}{d t} m_{N}^{2}= & 6 \lambda^{2}\left(m_{u}^{2}+m_{d}^{2}+m_{N}^{2}+A_{\lambda}^{2}\right)+4 \kappa^{2}\left(3 m_{N}^{2}+A_{\kappa}^{2}\right) \\
\frac{d}{d t} m_{\Psi_{Q}}^{2}= & 2 y_{1}^{2}\left(m_{U}^{2}+m_{\Psi_{Q}}^{2}+m_{T^{c}}^{2}+A_{y_{1}}^{2}\right)+2 y_{2}^{2}\left(m_{u}^{2}+m_{\Psi_{Q}}^{2}+m_{t^{c}}^{2}+A_{y_{2}}^{2}\right) \\
& -\frac{32}{3} g_{C}^{2} M_{C}^{2}-\frac{32}{3} g_{W}^{2} M_{W}^{2}-\frac{8}{9} g_{X}^{2} M_{X}^{2} \\
\frac{d}{d t} m_{t^{c}}^{2}= & 6 y_{2}^{2}\left(m_{u}^{2}+m_{\Psi_{Q}}^{2}+m_{t^{c}}^{2}+A_{y_{2}}^{2}\right)-\frac{32}{3} g_{C}^{2} M_{C}^{2}-\frac{32}{9} g_{X}^{2} M_{X}^{2} \\
\frac{d}{d t} m_{T^{c}}^{2}= & 6 y_{1}^{2}\left(m_{U}^{2}+m_{\Psi_{Q}}^{2}+m_{T^{c}}^{2}+A_{y_{1}}^{2}\right)-\frac{32}{3} g_{C}^{2} M_{C}^{2}-\frac{32}{9} g_{X}^{2} M_{X}^{2} .
\end{aligned}
$$

\section{B Values of soft terms at the messenger scale}

In this appendix, we give the boundary conditions for the soft terms in Eq. (A.2) at the messenger scale $M$.

$$
\begin{aligned}
m_{U}^{2} & =m_{D}^{2}=\frac{1}{\left(16 \pi^{2}\right)^{2}}\left(\frac{8}{3} g_{W}^{4}+\frac{8}{27} g_{X}^{4}\right) \Lambda^{2} \\
m_{u}^{2} & =m_{d}^{2}=\frac{1}{\left(16 \pi^{2}\right)^{2}}\left(\frac{8}{3} g_{W}^{4}+\frac{8}{27} g_{X}^{4}-6 \lambda^{2} \xi^{2}\right) \Lambda^{2}, \\
m_{N^{\prime}}^{2} & =0 \\
m_{N}^{2} & =\frac{1}{\left(16 \pi^{2}\right)^{2}}\left(48 \xi^{4}-24 \kappa^{2} \xi^{2}-16 g_{C}^{2} \xi^{2}-16 g_{W}^{2} \xi^{2}-\frac{8}{3} g_{X}^{2} \xi^{2}\right) \Lambda^{2} \\
m_{\Psi_{Q}}^{2} & =\frac{1}{\left(16 \pi^{2}\right)^{2}}\left(\frac{8}{3} g_{C}^{4}+\frac{8}{3} g_{W}^{4}+\frac{8}{27} g_{X}^{4}\right) \Lambda^{2}, \\
m_{t^{c}}^{2} & =\frac{1}{\left(16 \pi^{2}\right)^{2}}\left(\frac{8}{3} g_{C}^{4}+\frac{34}{27} g_{X}^{4}\right) \Lambda^{2} \\
m_{T^{c}}^{2} & =\frac{1}{\left(16 \pi^{2}\right)^{2}}\left(\frac{8}{3} g_{C}^{4}+\frac{34}{27} g_{X}^{4}\right) \Lambda^{2}, \\
A_{\lambda} & =\frac{A_{\kappa}}{3}=-\frac{6 \xi^{2}}{16 \pi^{2}} \Lambda \\
A_{\lambda^{\prime}} & =A_{y_{1}}=A_{y_{2}}=0 .
\end{aligned}
$$

\section{Renormalization Group Equations for "split" superpotential couplings}

Below the $S U(3)_{W}$ breaking scale $F$, the $S U(3)_{2}$-invariant superpotential couplings $\lambda$ and $y_{2}$ are split into separate couplings for the doublet and singlet components of the Higgs triplets $\mathcal{H}_{u}$ and $\mathcal{H}_{d}$ :

$$
W \ni \lambda_{s} N S_{u} S_{d}+\lambda_{d} N H_{u} H_{d}+y_{2, s} S_{u} T t^{c}+y_{2, d} H_{u} Q t^{c} .
$$


The associated RGEs, valid below the scale $F$, are given by:

$$
\begin{aligned}
\frac{d}{d t} \lambda_{s} & =\lambda_{s}\left(3 y_{2, s}^{2}+3 \lambda_{s}^{2}+2 \lambda_{d}^{2}+2 \kappa^{2}\right) \\
\frac{d}{d t} \lambda_{d} & =\lambda_{d}\left(3 y_{2, d}^{2}+\lambda_{s}^{2}+4 \lambda_{d}^{2}+2 \kappa^{2}-2\left(\frac{1}{2} g^{\prime 2}+\frac{3}{2} g^{2}\right)\right), \\
\frac{d}{d t} y_{2, s} & =y_{2, s}\left(5 y_{2, s}^{2}+2 y_{2, d}^{2}+\lambda_{s}^{2}-2\left(\frac{8}{9} g^{\prime 2}+\frac{8}{3} g_{C}^{2}\right)\right), \\
\frac{d}{d t} y_{2, d} & =y_{2, d}\left(y_{2, s}^{2}+6 y_{2, d}^{2}+\lambda_{d}^{2}-2\left(\frac{13}{18} g^{\prime 2}+\frac{3}{2} g^{2}+\frac{8}{3} g_{C}^{2}\right)\right),
\end{aligned}
$$

where $g_{C}, g$ and $g^{\prime}$ are the $S U(3)_{C}, S U(2)_{L}$ and $U(1)_{Y}$ gauge couplings, respectively, and $t \equiv\left(1 / 16 \pi^{2}\right) \ln \mu$. The matching conditions between the $S U(3)_{W} \times U(1)_{X}$ and $S U(2)_{L} \times U(1)_{Y}$ gauge couplings at the scale $F$ read:

$$
g=g_{W}, \quad g^{\prime}=\frac{g_{W} g_{X}}{\sqrt{g_{W}^{2}+g_{X}^{2} / 3}} .
$$

For completeness, we also give the matching conditions for the gaugino masses:

$$
M_{2}=M_{W}, \quad M_{1}=\frac{g_{X}^{2} M_{W}+3 g_{W}^{2} M_{X}}{3 g_{W}^{2}+g_{X}^{2}} .
$$

\section{References}

[1] L. Alvarez-Gaume, M. Claudson and M. B. Wise, Nucl. Phys. B 207 (1982) 96; M. Dine and W. Fischler, Phys. Lett. B 110 (1982) 227; C. R. Nappi and B. A. Ovrut, Phys. Lett. B 113 (1982) 175; S. Dimopoulos and S. Raby, Nucl. Phys. B 219 (1983) 479; M. Dine, A. E. Nelson and Y. Shirman, Phys. Rev. D 51 (1995) 1362 arXiv:hep-ph/9408384; M. Dine, A. E. Nelson, Y. Nir and Y. Shirman, Phys. Rev. D 53 (1996) 2658 arXiv:hep-ph/9507378. For a review, see G. F. Giudice and R. Rattazzi, Phys. Rept. 322 (1999) 419 [arXiv:hep-ph/9801271].

[2] G. R. Dvali, G. F. Giudice and A. Pomarol, Nucl. Phys. B 478 (1996) 31 arXiv:hep-ph/9603238].

[3] N. Arkani-Hamed, A. G. Cohen and H. Georgi, Phys. Lett. B 513 (2001) 232 arXiv:hep-ph/0105239; N N. Arkani-Hamed, A. G. Cohen, E. Katz, A. E. Nelson, T. Gregoire and J. G. Wacker, JHEP 0208 (2002) 021 arXiv:hep-ph/0206020]; N. Arkani-Hamed, A. G. Cohen, E. Katz and A. E. Nelson, JHEP 0207 (2002) 034 |arXiv:hep-ph/0206021]. For a review, see M. Schmaltz and D. Tucker-Smith, Ann. Rev. Nucl. Part. Sci. 55 (2005) 229 arXiv:hep-ph/0502182.

[4] P. H. Chankowski, A. Falkowski, S. Pokorski and J. Wagner, Phys. Lett. B 598 (2004) 252 arXiv:hep-ph/0407242]; A. Birkedal, Z. Chacko and M. K. Gaillard, JHEP 0410 (2004) 036 [arXiv:hep-ph/0404197).

[5] Z. Berezhiani, P. H. Chankowski, A. Falkowski and S. Pokorski, Phys. Rev. Lett. 96 (2006) 031801 |arXiv:hep-ph/0509311].

[6] T. S. Roy and M. Schmaltz, JHEP 0601 (2006) 149 [arXiv:hep-ph/0509357.

[7] C. Csaki, G. Marandella, Y. Shirman and A. Strumia, Phys. Rev. D 73 (2006) 035006 arXiv:hep-ph/0510294. 
[8] M. Schmaltz, JHEP 0408 (2004) 056 arXiv:hep-ph/0407143].

[9] H. P. Nilles, M. Srednicki and D. Wyler, Phys. Lett. B 120 (1983) 346; J. P. Derendinger and C. A. Savoy, Nucl. Phys. B 237 (1984) 307. For a review, see U. Ellwanger, C. Hugonie and A. M. Teixeira, arXiv:0910.1785.

[10] H. E. Haber and Y. Nir, Phys. Lett. B 306 (1993) 327 [arXiv:hep-ph/9302228]; H. E. Haber, arXiv:hep-ph/9505240.

[11] B. Bellazzini, C. Csaki, A. Falkowski and A. Weiler, Phys. Rev. D 80 (2009) 075008 arXiv:0906.3026 [hep-ph]].

[12] B. Bellazzini, C. Csaki, A. Falkowski and A. Weiler, Phys. Rev. D 81 (2010) 075017 arXiv:0910.3210 [hep-ph]].

[13] G. F. Giudice and R. Rattazzi, Nucl. Phys. B 511 (1998) 25 [arXiv:hep-ph/9706540].

[14] U. Ellwanger, Phys. Lett. B 349 (1995) 57 arXiv:hep-ph/9501227.

[15] U. Ellwanger, C. C. Jean-Louis and A. M. Teixeira, JHEP 0805 (2008) 044 arXiv:0803.2962 [hep-ph]].

[16] A. Delgado, G. F. Giudice and P. Slavich, Phys. Lett. B 653 (2007) 424 arXiv:0706.3873 [hep-ph]].

[17] A. Pomarol and S. Dimopoulos, Nucl. Phys. B 453 (1995) 83 arXiv:hep-ph/9505302;

R. Rattazzi, Phys. Lett. B 375 (1996) 181 [arXiv:hep-ph/9507315]. 